\title{
Shadow Detection Approach Combining Spectral and Geometrical Properties in Highway Video-Surveillance
}

\author{
Hakima Asaidi \\ Laboratory LIIAN \\ Department of Informatics \\ Faculty of sciences, Fez, \\ Morocco
}

\author{
Abdellah Aarab \\ Laboratory LESSI \\ Department of Physics \\ Faculty of sciences, Fez, \\ Morocco
}

\author{
Mohamed Bellouki \\ Department of Mathematics \\ and Informatics \\ Multidisciplinary Faculty, \\ Nador, Morocco
}

\begin{abstract}
In applications requiring objects extraction, cast shadows induce shape distortions and object fusions interfering performance of high level algorithms in video surveillance system. Shadow elimination allows to improve the performances of video object extraction, tracking and description tools. In this work, an approach to automatic shadow detection and extraction is proposed, which operates multiple properties derived from spectral, geometric and temporal analysis of shadows. A generic model that chooses the candidate shadow regions based on shadow direction is developed. Then, the validity of detected regions as shadows is verified using the capability of approach that allows associating to each photometric pixel its equivalent part of the shadow, while integrating the various parameters related to illumination and the surface. Simulation results show that the proposed approach is robust and efficient in detecting shadows for different background and changeable illumination conditions.
\end{abstract}

\section{General Terms}

Computer vision, Video surveillance system, Vehicles recognition and classification, Shadow removing.

\section{Keywords}

Visual surveillance, adaptive background subtraction, object extraction, shadow detection

\section{INTRODUCTION}

As the human vision cannot be complete without the intervention of the brain that interprets the information received from the eye and analyzes the different situations in an obvious manner, computer vision also cannot be completed without an intelligent vision system able to perform in a manner close to that of humans. The human eye can easily understand the perspective effects, overlapping and shadow, however the artificial vision does not distinguish the object of his shadow and considers both as a single moving object, which affects object recognition and its tracking. Despite this, in applications requiring the identification of objects, shadows modify the perceived shape and color, thus introducing a distortion in the object detection process. For this reason, the problem of shadow detection and elimination is a great challenge and a lot of researches have been proposed to characterize shadows for their extraction [1]-[16].

A shadow is a dark area created by the interposition of an opaque object between a light source and the surface on which the light is reflected. There are two different types of shadows: projected shadow is cast by an object on a surface which belongs to a different object is called cast shadow or extrinsic shadow. Self shadow is an intrinsic shadow because it is cast by an object on itself, on the part opposite the light. And a shadow can be Umbra (dark shadow), Penumbra (soft shadow), or both.

Shadow detection approaches are either colorimetric propertybased or geometric property-based. Colorimetric propertybased approaches use features like color, brightness, texture or boundaries to identify shadowed regions. Geometric propertybased techniques need information of shape and position of the moving object and light source. Several researchers have modeled shadow in the physical sense, they describe shadow as the product of the irradiance and reflectance of the object surface such as the work presented in [1], the authors exploit information of static edge, uniform changes of shading and penumbra of shadow, obtained by color ratio and difference, to detect background regions which are covered or uncovered by a moving cast shadow. The same model with the novelty of using TSV color space, the authors try to improve the approach [2]. The detection is based on the observation that shadows change significantly the brightness of an area without significantly modifying the color information. Another approach exploit texture property to detect moving shadow is presented in [3], they authors are based on the assumption that shadow regions present same textural characteristics in each frame and in the corresponding adaptive background. And they compare texture descriptors for foreground pixels that are darker and their corresponding in background. Those approaches and others assume that the value of the surface under cast shadows will be linearly attenuated from the background value. Some recent works contradicts this hypothesis of linearity [4], the authors consider that the assumption will falsely labels pixels as cast shadows when foreground objects have chromaticity values similar to that of the background, and they propose an approach which allows differentiation of foreground and cast shadow values with similar chromaticity. This non proportionality is addressed also in [5] via a dichromatic reflection model, this method performs a segmentation based on blue-ratio and spatio-temporal albedo test, and making no assumptions about geometry of the scene but requiring a static background.

Others approaches combine photometric and geometric property of shadows such as the work proposed in [6], where a $2 \mathrm{D}$ joint vehicle-shadow model was proposed to obtain the vehicle and the shadow location from luminance analysis and lighting directions. In the work [7], the authors first suppose the presence of shadow based on fact that shadow darkens the surface, after they validate the hypothesis using geometric property of shadow and photometric color invariants that describe the color configuration of each image point discounting shadows and highlights. In [8], the authors are based on the same hypothesis of shadow spectral properties 
presented in [7], and proposed to segment the boundary between self-shadow and cast shadow, they define an occluding function by exploiting geometrical property of shadow, and the feature points of occluding function are detected using multi-resolution wavelet transform.

Another proposed approach of cast shadows elimination are neither spectral property based nor geometric property based [9], the authors use the lane dividing lines for removing shadows, and propose an automatic method for detecting lane dividing lines. They suppose that the detected vehicle (moving region) moves close to one of lane centers and they construct a vehicle histogram by accumulating all positions of detected vehicles, for estimating all desired lane centers. However, it is obvious that the detection of lanes is not accurate because it is based on the centroids of moving objects that contain the vehicle and shadow, and therefore the elimination of the shadow will not be exact. Further, this method cannot eliminate shadows that do not cross the lines.

Statistical approaches are also used to model cast shadow such as the works presented in [10] and [11]. Statistical learning techniques are used to capture the surface appearance under cast shadows, employing Gaussian mixture models (GMM). Recently, in [12] an adaptive shadow estimator is proposed to detect and eliminate the shadow of a moving object by automatically adapting to the variation in illumination and environment. The proposed method discriminates between the shadow and the moving object by cascading three estimators; chromaticity difference, brightness difference, and local relation.

In [13], first, candidate shadow region and a corresponding region are segmented by setting gray level thresholds, and neighbor-matching regions are constructed with a mathematical morphological algorithm. Based on hypothesis that corresponding shadow region and non-shadow region, in the same scene, share similar gray level features after linear transformation. Gray level distribution functions of the region are constructed and Kolmogorov tests are used to test the distribution equality of gray levels in the two regions. Shadow regions are obtained by selecting the region with relatively lower average gray level from the matched region pair. The particle swarm optimization (PSO) algorithm is used to facilitate the feature extraction during the matching process. But the selection of candidate shadow region is not accurate because the spatial information is not sufficiently incorporated. [14] presented a background subtraction and shadow removal algorithm for processing grey scale video sequences, using a statistical approach combined with geometrical constraints. In this algorithm, a background model using a metrically trimmed mean is extracted in a training stage, and foreground pixels are obtained in the operation (or test) stage. The statistical model is combined with expected geometrical properties for shadow identification and removal. Finally, morphological operators are applied, to remove isolated foreground pixels. [15] proposed an algorithm for removing shadows by combining texture and statistical models. They use a Gaussian Mixture Model for background removal and the detection of moving shadows in test images. They also define two indices for characterizing non-shadowed regions; one of which indicates the characteristics of edges and the other is characterised by the information in gray scales of images used for building modified darkening factors based on Gaussian models.

We can report to [16], which present a survey of moving cast shadow detection approaches and perform a comparative study between approaches in term of quantitative performance measures and qualitative observations.

In this paper, an approach to automatic shadow detection is proposed, which operates multiple properties derived from spectral, geometric and temporal analysis of shadows. A generic model that detects different candidate shadow regions has developed, using time and shadow direction. And verifies the hypothesis of shadow by using the color analysis. The approach allows associating to each photometric point its equivalent part of the shadow, while integrating the various parameters related to illumination and the surface. The rest of this paper is organized as follows. In the next section, the overview of the whole proposed approach is described. Section 3 reports experimental results. Finally, Section 4 concluded this paper.

\section{OVERVIEW OF THE PROPOSED APPROACH}

The proposed approach is summarized in Fig. 1.

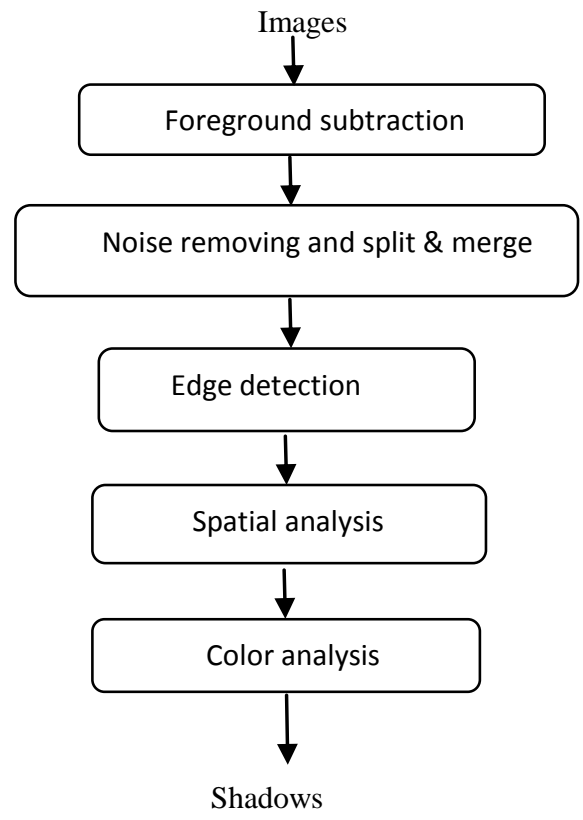

Fig 1: Framework of shadow detection and extraction

First of all, moving objects have being identified by using a robust and automatic segmentation approach based on the adaptive background subtraction scheme proposed in [3], because it allow to take account the sudden changes in illumination and other scene parameters that alter the appearance of the background. Second, a series of simple morphological operations is used for noise removal and the algorithm of split and merge is applied, for eliminating regions if their size is small and merging any two adjacent regions if they are very close to each other. A result of these steps is a number of objects but with their shadows. To detect and eliminate shadow, we pass to the main proposed part that is organized in two levels: spatial analysis and color analysis. Spatial analysis consists of determining candidate shadow regions. We propose to exploit geometric properties of shadows related to shadow direction and object boundaries. The direction of shadow depends on the relative locations of the light source, the object, and the camera. Assume that the camera is static. And determine the direction of shadow according to the location of sun. The shadow of the object rotates during the day while changing in length. In the 
morning the shadow is long and oriented towards southwest, in the evening shadow is long and oriented towards the southeast. As a result, the shadow is the shortest in the middle of the day (around 13h in winter, so $14 \mathrm{~h}$ in summer at noon solar time). At solar noon, the shadow is minimal but not zero. Known direction of the shadow, the candidate shadow region can be chosen. In the same direction, there exists a light region in corresponding background that has same textural characteristics but different color, called in this paper candidate antecedent region. The hypothesized shadow region is then verified by the analysis of spectral property. Color analysis consists of associating to candidate antecedent region its equivalent part of the shadow, based on the assumption that the mapping from light region to shadowed region is linear. And the similarity between obtained region and candidate shadow region is then evaluated. Finally the initial hypothesis is accepted or rejected. In the following sections, the different stages of the proposed approach are presented in detail.

\subsection{Spatial Analysis}

The shadow is projected onto a region near the object and in certain direction. According to time of day, eight directions (as shown in Fig. 2) correspond to eight time intervals is considered. These intervals are obtained by dividing the duration of daylight by 8 .

In general, the length of day varies throughout a year and depends on the latitude. This variation is caused by tilting the axis of rotation of the Earth relative to the ecliptic plane. It is the shortest at the December solstice in the northern hemisphere and June in the southern hemisphere, the longest in the June in the southern hemisphere and December in the southern hemisphere, during the equinoxes, day length is on average 12 hours over the entire Earth. Day length is calculated according to:

- The latitude of the site in question: $\lambda$

- Date: angle $\delta$ traveled by the earth in its orbit, counted from its position at the vernal equinox.

- $\quad$ The latitude of the tropics: $\alpha=23^{\circ} 27^{\prime}$.

The formula for the length of daylight hours is as follows:

$$
D=12 \gamma / \pi
$$

where

$$
\gamma=-2 \operatorname{Arccos}(\tan \lambda \times \tan (\operatorname{Arcsin}(\sin \alpha \times \sin \delta))) .))
$$

Known the longitude, latitude and time zone in the region, the duration of daylight can be calculated from formula (1).

\begin{tabular}{|c|c|c|}
\hline 1 & 4 & 6 \\
\hline 2 & Obj & 7 \\
\hline 3 & 5 & 8 \\
\hline
\end{tabular}

Fig 2: Object with its shadow and the eight possible shadow directions

Let us refer to the object under analysis with $I(x, y)=(R(x, y)$, $G(x, y), B(x, y))$, where $R, G$ and $B$ represent the three color channels and $(x, y)$ indicates a generic pixel position. In order to obtain the edge of different regions in the object, the Sobel method is applied that finds edges using the Sobel approximation to the gradient image. The pixels edge are the pixels correspond to local maxima of the gradient intensity.
Applying the Sobel detector separately on the three color channels, a pixel is defined as a pixel edge if the corresponding magnitude of the maximum of the gradient of the three color components exceeds a certain threshold. The choice of threshold depends on the image characteristics, a low value of threshold allows to detect small edge and a high value of threshold allows to detect strong edge. In the experiments, the threshold is determined empirically.

According to the time of day, the direction of shadow is determined and, in this direction, the extreme region in the object is chosen as a candidate shadow region Sc.

Let us consider $P_{(x, y)}(a, b, c)$ a pixel in Sc, where $a, b$ and $c$ are the intensity value for the components $\mathrm{R}, \mathrm{G}$ and $B$ respectively. And the reference pixel $P_{(x r, y r)}\left(a^{\prime}, b^{\prime}, c^{\prime}\right)$ is a neighbor of the pixel under analysis and it belongs to the light region in corresponding background. It is defined as:

$\left(x_{r}, y_{r}\right)=(x+\alpha, y+\beta)$ where $\alpha, \beta \in\{-\boldsymbol{\epsilon}, 0, \boldsymbol{\epsilon}\}$ and $\left(x_{r}, y_{r}\right) \neq(x$, $y)$, i.e., $\alpha$ and $\beta$ are not simultaneously equal to zero. According to direction of shadow, $\alpha$ and $\beta$ can be determined.

\subsection{Color Analysis}

The identification of a set of pixels, Sc, which are candidates to be shadow pixels, is performed in preview section.

Giving a color vector of a pixel in antecedent candidate region, it follows that each of the three RGB color components, decrease when passing from a lit region to a shadowed one. This variation is more or less severe as a function of time of day. In the proposed model, these variations in terms of illumination are solved through the formulation of the problem and a real-time calculation of certain parameters. Assume that the region is homogeneous, this assumption is often verified in highways, our application of interest. Then, proceed to a verification of color characteristics. Let us consider a real vectors space $E$, where $E$ represent the RGB color space. Let $f_{t}$ denote a function from $E$ to $E$, where $f_{t}$ is the mapping from light to shadow.

Let $P_{(x r, y r)}\left(a^{\prime}, b^{\prime}, c^{\prime}\right)$ be a vector of $E . P$ can be written as:

$$
\begin{gathered}
P_{\left(x_{r}, y_{r}\right)}\left(a^{\prime}, b^{\prime}, c^{\prime}\right)=a^{\prime} \times R+b^{\prime} \times G+c^{\prime} \times B \\
\left.f_{t}\left(P_{r}\right)=f_{t}\left(a^{\prime} \times R+b^{\prime} \times G+c^{\prime} \times B\right) . B\right)
\end{gathered}
$$

Assume that $f_{t}$ is a linear function. The following equality holds:

$$
\left.f_{t}\left(P_{r}\right)=a^{\prime} x f_{t}(R)+b^{\prime} x f_{t}(G)+c^{\prime} x f_{t}(B) .\right) .(5)
$$

The calculation of the shadow of the three components $\mathrm{R}, \mathrm{G}$ and $\mathrm{B}$ is real-time. (5) becomes as follow,

$$
\boldsymbol{f}_{t}\left(\boldsymbol{P}_{r}\right)=\boldsymbol{P},
$$

A large amount of randomly generated references pixels is used and the intensity of each generated pixel $P_{(x r, y r)}$ and its neighborhood pixel in the Sc, candidate shadow region, is compared. A Euclidean distance is used and a threshold is applied to the result. A pixel is classified as shadow if the corresponding two coefficients vectors color present similar intensity, or are close. Finally, the initial hypothesis is confirmed or rejected for every detected region.

\section{EXPERIMETAL RESULTS}

In order to analyze the performance of the proposed approach, several experiments have been carried out on different test images. A selection of test images is shown in Fig. 3. At the beginning, different objects are extracted from the analyzed 
image through adaptive background subtraction as shown is Fig. 4. After that, the edges of objects are extracted by applying the Sobel operator, to detect different regions in the object. Fig. 5 shows the result of edge detection. To determine shadow direction, first the duration of daylight is determined using (1) as shown in the following table.

Table 1. The number of sunny hours per day during the first week of January in Fez city-Morocco.

\begin{tabular}{|l|l|l|l|l|l|l|l|}
\hline $\begin{array}{l}\text { Number } \\
\text { of day }\end{array}$ & 01 & 02 & 03 & 04 & 05 & 06 & 07 \\
\hline $\begin{array}{l}\text { Duration } \\
\text { of } \\
\text { daylight }\end{array}$ & 09.56 & 09.57 & 09.58 & 09.59 & 09.59 & 10.00 & 10.01 \\
\hline
\end{tabular}

After that, the duration of daylight is divided by eight to obtain the time intervals. Known the object location and the time interval, shadow direction is determined, which is one or more of the eight directions. According to the result of edge detection and the direction of shadow, the candidate shadow region is chosen as shown in Fig. 6. To confirm the presence of shadow, we proceed to color analysis. In the direction of shadow, an amount of references pixels is randomly chosen and these values using the equation (5) are calculated. The values of $R, G$ and $B$ under shadow are obtained empirically. Finally, the values of pixels in candidate shadow region and their neighborhoods are compared. Fig. 7 shows that shadow are correctly detected by the proposed approach in different background and changeable illumination conditions.

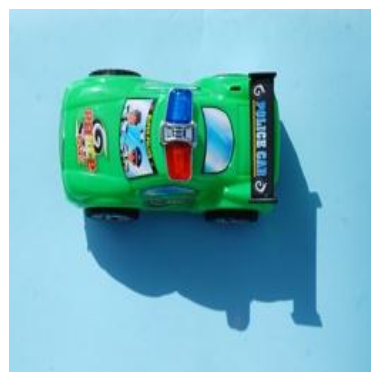

(a) Current image

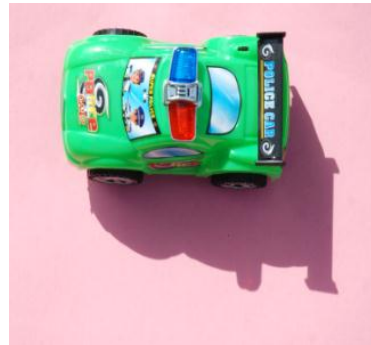

(c) Current image

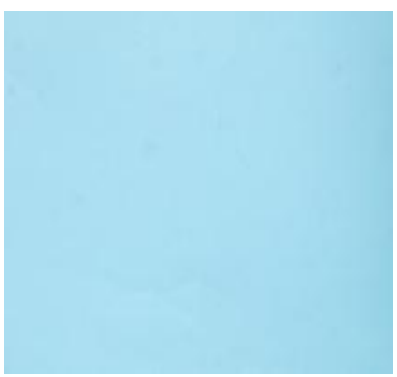

(b) Background image

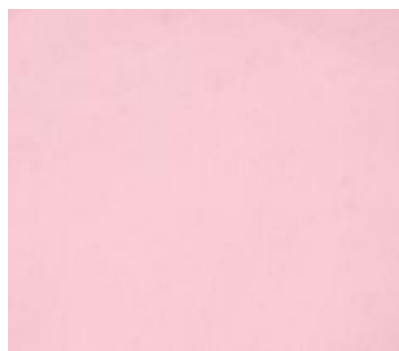

(d) Background image
Fig 3: Examples of frames (a, c) and background models $(b, d)$ in different color

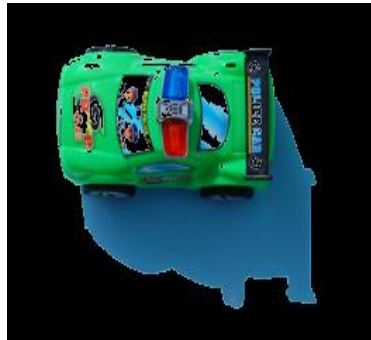

(a)

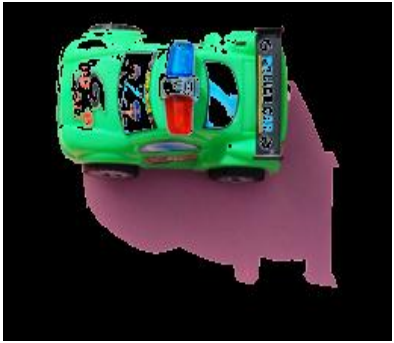

(b)
Fig 4: Results of background subtraction

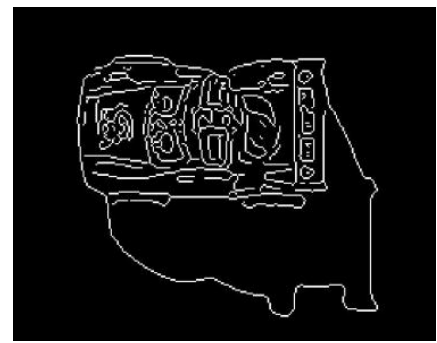

Fig 5: Result of edge detection

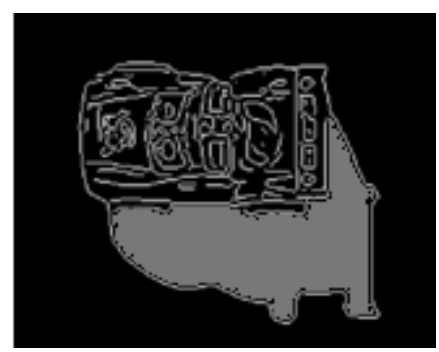

Fig 6: Candidate shadow region (gray pixels)

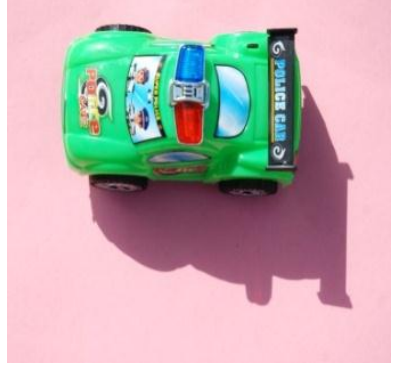

(a)

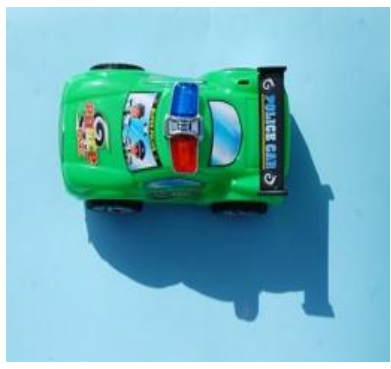

(c)

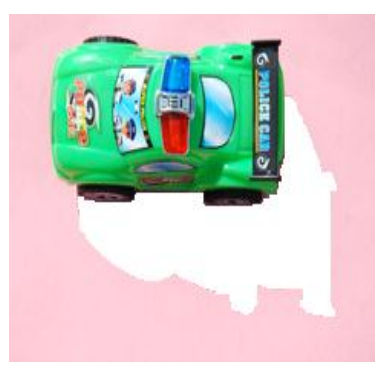

(b)

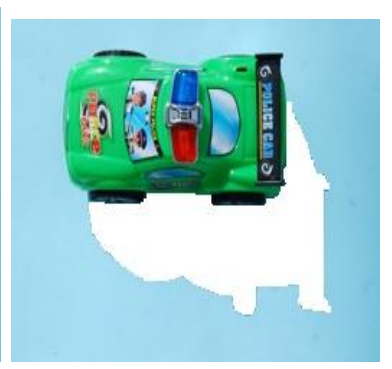

(d)
Fig 7: Shadow detection results for the test images, (a, c) original image, $(b, d)$ shadow (white pixels) 


\section{CONCLUSION}

In this paper, we have presented an approach for shadow removal by combining geometric and colorimetric analysis. The candidate shadow region is determined using spatial and temporal analysis of shadow direction. The spatial analysis is not based on any a priori assumptions about the scene geometry or shape of the object to be extracted. Therefore, the approach is general and can be used for a wide range of applications with different data types. The colorimetric analysis is also used to improve the presence of shadow. The evaluation of the approach was made through a number of videos representing real environments. The results confirmed the validity of the approach, including its ability to cope with varied content and its robustness against luminance changes.

\section{ACKNOWLEDGMENTS}

The present work is supported by the CNRST "Centre National de la Recherche Scientifique et Technique" of Morocco under Grant $\mathrm{N}^{\circ}: \mathrm{E} 10 / 001$

\section{REFERENCES}

[1] Stauder,J., Mech, R. and Ostermann, J. 1999. Detection of moving cast shadows for object segmentation. IEEE Trans. Multimedia, 1, (1), 65-77.

[2] Cucchiara, R., Grana, C., Piccardi, M., Prati, A. and Sirotti, S. 2001. Improving shadow suppression in moving object detection with HSV color information. Proc. IEEE Int. Cof. Intelligent Transportation Systems (ITSC '01), Oakland, Calif, USA, 334-339.

[3] Leone, A., Distante, C. 2007. Shadow detection for moving objects based on texture analysis. Pattern Recognition , 40, 1222 - 1233.

[4] Martel-Brisson, N. and Zaccarin, A. 2008. Kernel-based learning of cast shadows from a physical model of light sources and surfaces for low-level segmentation. In Proc. Conf. Computer Vision and Pattern Recognition., 1-8.

[5] Nadimi, S., Bhanu, B. 2004. Physical models for moving shadow and object detection in video, IEEE Trans. Pattern. Anal. Mach. Intell., 26, (8), 1079-1087.

[6] Yoneyama, A., Yeh, C.-H. and Jay Kuo, C.-C. 2005. Robust vehicle and traffic information extraction for highway surveillance. EURASIP J. App. Signal Process., $14,2305-2321$.
[7] Salvador, E. Cavallaro, A. and Ebrahim, T. 2004. Cast shadow segmentation using invariant color features. Computer Vision and Image Understanding, 95, 238259.

[8] Fang, L. Z., Qiong, W. Y., Sheng, Y. Z. 2008. A method to segment moving vehicle cast shadow based on wavelet transform. Pattern Recognition Letters, 29, 2182-2188.

[9] Hsieh, J.-W., Yu, S.-H., Chen, Y.-S. and Hu, W.-F. 2006. An automatic traffic surveillance system for vehicle tracking and classification. IEEE Transactions on Intelligent Transportation Systems, vol. 7, no. 2, 175187.

[10] Liu, Z., Huang, K., Tan, T. and Wang, L. 2007. Cast shadow removal combining local and global features. In Proc. CVPR Workshop 7th Int.Workshop VS, 1-8.

[11] Martel-Brisson, N. and Zaccarin, A. 2007. Learning and removing cast shadows through a multidistribution approach. IEEE Trans. Pattern. Anal. Mach. Intell., vol. 29, no. 7, 1133-1146.

[12] Choi, J. M., Yoo, Y. J., Choi, J. Y. 2010. Adaptive shadow estimator for removing shadow of moving object. Computer Vision and Image Understanding, 114, 1017-1029.

[13] Chao, X., Yanjun, L., Ke, Z., Ling, W. 2011. Shadow detecting using particle swarm optimization and the Kolmogorov test. Computers and Mathematics with Applications, 62, 2704-2711.

[14] Jung, C. R. 2009. Efficient Background Subtraction and Shadow Removal for Monochromatic Video Sequences. IEEE Transactions on Multimedia, 11, (3), 571-577.

[15] Lin, C.-T., Yang, C.-T., Shou, Y.-W., Shen, T.-K. 2010. An Efficient and Robust Moving Shadow Removal Algorithm and Its Applications in ITS. EURASIP Journal on Advances in Signal Processing (945130), 119.

[16] Sanin, A., Sanderson, C., Lovell, B. C. 2012. Shadow detection: A survey and comparative evaluation of recent methods. Pattern Recognition, 45, 1684-1695. 